\title{
Multiscale finite element calculations in Python using SfePy
}

\author{
R. Cimrman ${ }^{\mathrm{a}}$, V. Lukeš ${ }^{\mathrm{b}}$, E. Rohan ${ }^{\mathrm{b}}$ \\ ${ }^{a}$ New Technologies - Research Centre, University of West Bohemia, Univerzitni 8, \\ 301 00, Pilsen, Czech Republic \\ ${ }^{b}$ NTIS - New Technologies for the Information Society, Faculty of Applied Sciences, \\ University of West Bohemia, Univerzitni 8, 301 00, Pilsen, Czech Republic
}

\begin{abstract}
SfePy (Simple finite elements in Python) is a software for solving various kinds of problems described by partial differential equations in one, two or three spatial dimensions by the finite element method. Its source code is mostly (85\%) Python and relies on fast vectorized operations provided by the NumPy package. For a particular problem two interfaces can be used: a declarative application programming interface (API), where problem description/definition files (Python modules) are used to define a calculation, and an imperative API, that can be used for interactive commands, or in scripts and libraries. After outlining the SfePy package development, the paper introduces its implementation, structure and general features. The components for defining a partial differential equation are described using an example of a simple heat conduction problem. Specifically, the declarative API of SfePy is presented in the example. To illustrate one of SfePy's main assets, the framework for implementing complex multiscale models based on the theory of homogenization, an example of a two-scale piezoelastic model is presented, showing both the mathematical description of the problem and the corresponding code.
\end{abstract}

Keywords: finite element method, multiscale simulations, piezoelasticity, SfePy, Python

Email addresses: cimrman3@ntc.zcu.cz (R. Cimrman), vlukes@ntis.zcu.cz (V. Lukě̌), vlukes@ntis.zcu.cz (E. Rohan) 


\section{Introduction}

SfePy (http://sfepy.org) is a software for solving systems of coupled partial differential equations (PDEs) by the finite element method (FEM) in 1D, 2D and 3D. It can be viewed both as a black-box PDE solver, and as a Python package which can be used for building custom applications. It is a multi-platform (Linux, Mac OS X, Windows) software released under the New BSD license - the source code hosting, issue tracker and continuous integration tools are available thanks to the GitHub development platform.

SfePy has been employed by our group for a range of topics in multiscale modelling in biomechanics and materials science, including multiscale models of biological tissues (bone, muscle tissue with blood perfusion) $[9,38,10,34,41,40]$, a fish heart model with active contraction [27], computations of acoustic transmission coefficients across interfaces of arbitrary microstructure geometry [35], computations of phononic band gaps [39, 37], the finite element formulation of the Schroedinger equation $[47,12,11]$, and other applications.

In Section 2 the programming language choice is discussed and the SfePy project development is described. In Section 3 an overview of the package is given, and a simple example definition (a heat conduction problem) is presented. Section 4 introduces the SfePy's homogenization engine a sub-package for defining complex multiscale problems using a simple domain specific language, based on Python's dictionaries. Finally, in Section 5 a complex example - a multiscale numerical simulation of a piezoelectric structure - is shown as expressed in the homogenization engine syntax.

\section{Development}

The code has been written primarily in the Python programming language $^{1}$. Python is a very high-level interpreted programming language, that has a number of features appealing to scientists (non-IT), such as: a clean, easy-to-read syntax, no manual memory management, a huge standard library, a very good interoperability with other languages (C, fortran), and a large and friendly scientific computing community. It allows both fast exploration of various ideas and efficient implementation, thanks to many

\footnotetext{
${ }^{1}$ SfePy sources (version 2018.3) GitHub statistics: Python 85.1\%, C 14.6\%, other: 0.3 $\%$.
} 
high-performance solvers with a Python interface, and numerical tools and libraries available among open source packages.

There are many finite element packages, commercial or open source, that can be used from Python, and some of them use Python as a primary language, notably Fenics [2] or Firedrake [33]. This indicates viability of our choice and is in agreement with our positive experience with the language.

The SfePy project uses Git [20] for source code management and GitHub development platform [21] for the source code hosting and developer interaction, see https://github.com/sfepy/sfepy, similarly to many other scientific Python tools. Travis CI [46] is used (via GitHub) for running automatic tests after every uploaded commit. The developers and users of the software can communicate using the mailing list "sfepy@python.org". The source code and the package usage and development are documented with the help of the Sphinx documentation generator [45] that is also used to generate the pages of the SfePy project web-site.

The version 2018.3 has been released in September 17, 2018, and its git-controlled sources contained 897 total files, 721447 total lines (1539277 added, 817830 removed) and 6386 commits done by 24 authors $^{2}$. About 120000 lines $(16 \%)$ are the source code, the other lines belong to the finite element meshes, documentation etc.

\section{Description}

In this section we briefly outline the package implementation, structure and general features. The components for defining a PDE to solve are described using a simple example in Section 3.4.

\subsection{Performance due to scientific Python ecosystem}

Because Python is an interpreted language, and the standard implementation (CPython) has slow loops, several approaches are used in the code to achieve good (C-like) performance. For speed in general, it relies on fast vectorized operations provided by NumPy arrays [30], with significant use of advanced features such as broadcasting. C and Cython [6] are used in places where vectorization is not possible, or is too difficult/unreadable.

\footnotetext{
${ }^{2}$ Most of the authors contributed only one or a few commits.
} 
SfePy relies on a number of packages of the scientific Python software stack, namely: SciPy [25] for sparse matrices, solvers and algorithms, Matplotlib [24] for 2D plots, Mayavi [32] for 3D plots and simple post-processing GUI, PyTables [31] for HDF5 file format support, SymPy [29] for symbolic operations/code generation, igakit (a part of PetIGA [16]) for working with NURBS bases of the isogeometric analysis $[14,5]$ etc.

Besides the vectorized operations of NumPy, other performance gains are enabled by using very efficient solvers with a Python interface, such as UMFPACK [18] + scikit-umfpack [42], MUMPS [3] or PETSc [4]. SfePy can run in parallel, using PETSc + petsc4py [17] and mpi4py [15] packages. We employ the separation of concerns strategy with respect to parallelism: most of the SfePy code is serial, and there is a single dedicated module in SfePy, that, together with the flexible way of computing weak form integrals on any subdomain (see below), allows parallel assembling of the discrete systems and their subsequent solution.

\subsection{Design overview}

In $S f e P y$, the equations are not given in a fully symbolic way, as in, for example, Fenics or Firedrake projects ${ }^{3}$, but a simpler approach is used: the SfePy package comes with a database of predefined terms. A term is the smallest unit that can be used to build equations. It corresponds to a weak formulation integral over a (sub)domain and takes usually several arguments: (optional) material parameters, a single virtual (or test) function variable and zero or more state (or unknown) variables. The available terms are listed at our web site [43], currently there are 118 terms.

The high-level code that handles a PDE discretization in SfePy is independent of a method of domain or variable discretization. For each particular method of discretization, there is a sub-package that implements the specific functionality (degrees of freedom management, selection of subdomains, reference domain mappings, etc.). This abstraction allows adding various discretization methods. The following ones are currently implemented:

- the finite element method on 1D line, 2D area (triangle, rectangle) and 3D volume (tetrahedron, hexahedron) finite elements; with two kinds of polynomial bases:

\footnotetext{
${ }^{3}$ Both use the Unified Form Language from Fenics.
} 
- the classical nodal (Lagrange) basis that can be used with all supported element/cell types;

- the hierarchical (Lobatto) basis [44] that can be used with tensorproduct elements (line, rectangle, hexahedron).

The basis function polynomials of an arbitrary order (theoretically, see limitations below) as well as the corresponding quadrature rules are supported. The Lagrange basis is implemented in C/Cython, while the Lobatto basis functions use a C code generated using SymPy.

- the isogeometric analysis [14] with a NURBS or B-spline basis, implemented using the Bézier extraction approach [5], currently limited to single NURBS patch domains, see [7].

All the basis functions listed above support the $H^{1}$ function spaces only ( $\vec{H}$ (curl), $\vec{H}(\operatorname{div})$ spaces are not currently implemented). In addition to the above elements, two structural elements are implemented (using $\mathrm{SfePy}$ terms): the hyperelastic Mooney-Rivlin membrane [48], and the shell10x element term based on the Reissner-Mindlin theory [49].

\subsection{Working with SfePy}

$S f e P y$ can solve many problems described by PDEs in the weak form. For a particular problem, there are two interfaces that can be used:

- a declarative API, where problem description/definition files (Python modules) are used to define a calculation;

- an imperative API, that can be used for interactive commands, or in scripts and libraries.

Both the above APIs closely correspond to the mathematical description of the weak form PDEs. An advanced use of the declarative API is demonstrated in the piezoelectric model example in Section 5.

The declarative API involves almost no programming besides using basic Python data types (dicts, lists, tuples, strings, etc.) and allows a lazy definition of the problem, called problem configuration, as well as a manipulation with the problem configuration. Prior to a problem solution, the problem configuration is automatically translated into a problem object using the imperative API. The SfePy package contains several top-level scripts that can be used to run simulations defined using the declarative API. The two common ones are: 
- the simple.py script that allows running regular calculations of PDEs,

- the homogen.py script that allows running the homogenization engine to compute effective material parameters, see Section 4.

The imperative API allows immediate evaluation of expressions, and thus supports interactive exploration or inspection of the FE data. It is also more powerful than the declarative API as a user is free to perform non-predefined tasks. The problems defined using the imperative API usually have a main() function and can be run directly using the Python interpreter.

In the both cases, a problem definition is a Python module, so all the power of Python (and supporting SfePy modules) is available when needed for complex problems.

\subsection{Simple example: heat conduction}

Systems of PDEs are defined using keywords or classes corresponding to mathematical objects present in the weak formulation of the PDEs. Here we illustrate the components of the problem definition using a simple example. We wish to solve a heat conduction problem, that can be written in the weak form as follows: Find the temperature $u \in H^{1}(\Omega)$ such that for all $v \in H_{0}^{1}(\Omega)$ holds

$\int_{\Omega} v \frac{\partial u}{\partial t}+\int_{\Omega} c \nabla v \cdot \nabla u=0, \forall v, u(x, 0)=g(x), u(x, t)=\left\{\begin{array}{rl}-2 & x \in \Gamma_{\text {left }}, \\ 2 & x \in \Gamma_{\text {right }},\end{array}\right.$

where $c$ is a material parameter (a thermal diffusivity). Below we show the declarative way of defining the ingredients necessary to solve this problem with SfePy. For complete examples illustrating both the declarative and imperative APIs, see the accompanying dataset [8].

- The domain $\Omega$ has to be discretized, resulting in a finite element mesh. The mesh can be loaded from a file (generated by external tools) or generated by the code (simple shapes).

filename_mesh $=$ 'meshes/3d/cylinder.mesh'

- Regions serve as domains of integration and allow defining boundary and initial conditions. Subdomains of various topological dimension can be defined. The mesh/domain and region handling uses a $\mathrm{C}$ data structure adapted from [28]. The following code defines the domain $\Omega$ and the boundaries $\Gamma_{\text {left }}, \Gamma_{\text {right }}$. 


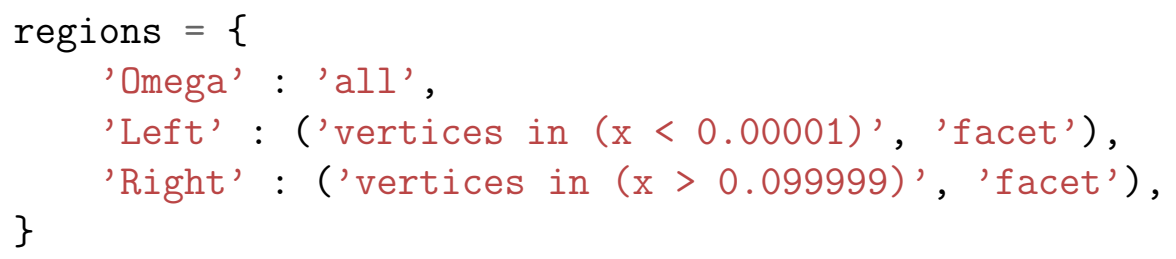

- Fields correspond to the discrete function spaces and are defined using the (numerical data type, number of components, region name, approximation order) tuple. A field can be defined on the whole domain, on a volume (cell) subdomain or on a surface (facet) region.

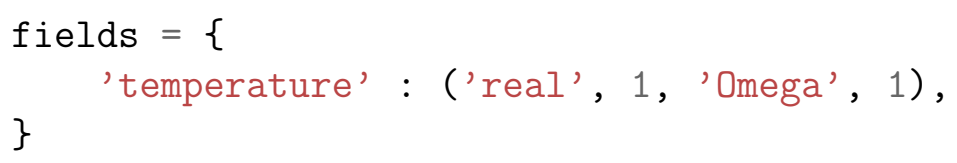

- The fields (FE spaces) can be used to define variables. Variables come in three flavors: unknown field for state variables, test field for test (virtual) variables and parameter field for variables with known values of degrees of freedom (DOFs). The definition items for an unknown variable definition are: ('unknown field', field name, order in global vector, [optional history size]). In the snippet below, the history size is 1 , as the previous time step state is required for the numerical time derivative. For a test variable, the last item is the name of the corresponding unknown variable.

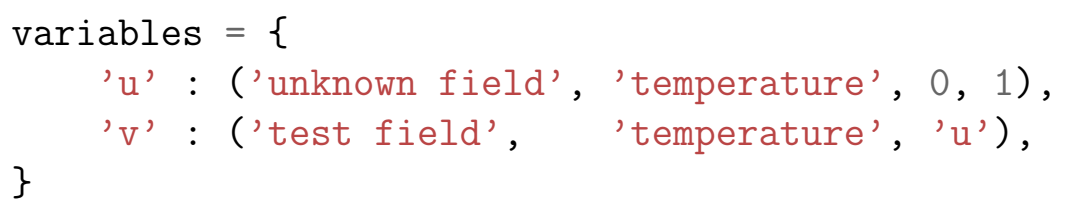

- Materials correspond to all parameters defined point-wise in quadrature points, that can be given either as constants, or as general functions of time and quadrature point coordinates. Here we just define the constant parameter $c$, as a part of the material ' $\mathrm{m}$ '.

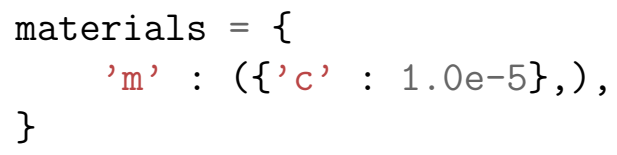


- Similarly to materials, the Dirichlet (essential) boundary conditions can be defined using constants or general functions of time and coordinates. In our case we set the values of $u$ to 2 and -2 on $\Gamma_{\text {left }}$ and $\Gamma_{\text {right }}$, respectively.

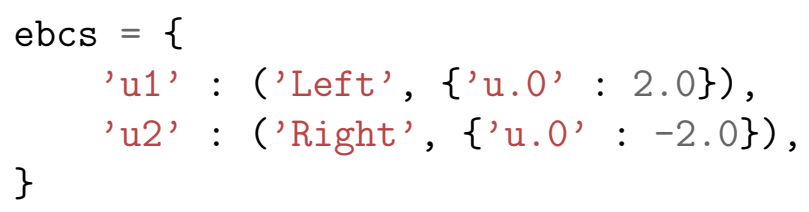

- The initial conditions can be defined analogously, here we illustrate how to use a function. The conditions are applied in the whole domain $\Omega$. The code assumes NumPy was imported (import numpy as $\mathrm{np}$ ), and ic_max is a constant defined outside the function.

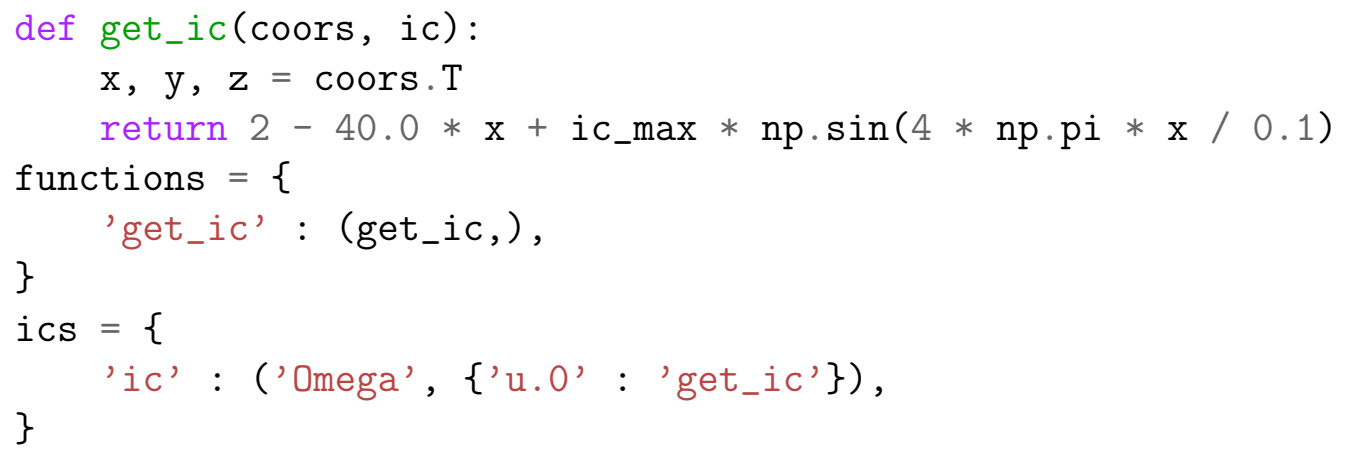

- The PDEs can be built as a linear combination of many predefined terms. Each term has its quadrature order and its region of integration. The integral specifies a numerical quadrature order.

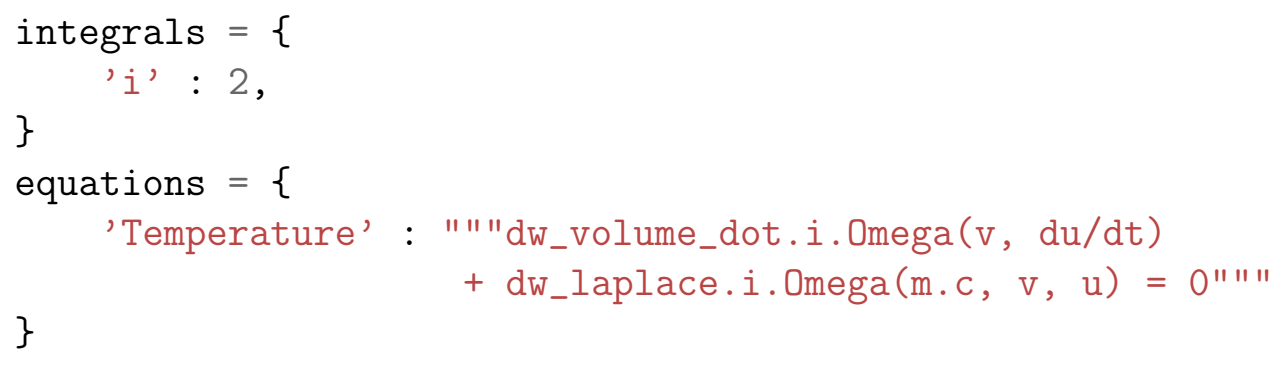

- A complete example contains additional information (notably a configuration of solvers), see [8]. There, the above code snippets are used in 
the heat_cond_declarative.py file. The simulation is then launched using the python <path/to/>simple.py heat_cond_declarative.py command. In Fig. 1, illustrative results of the above computation are shown.
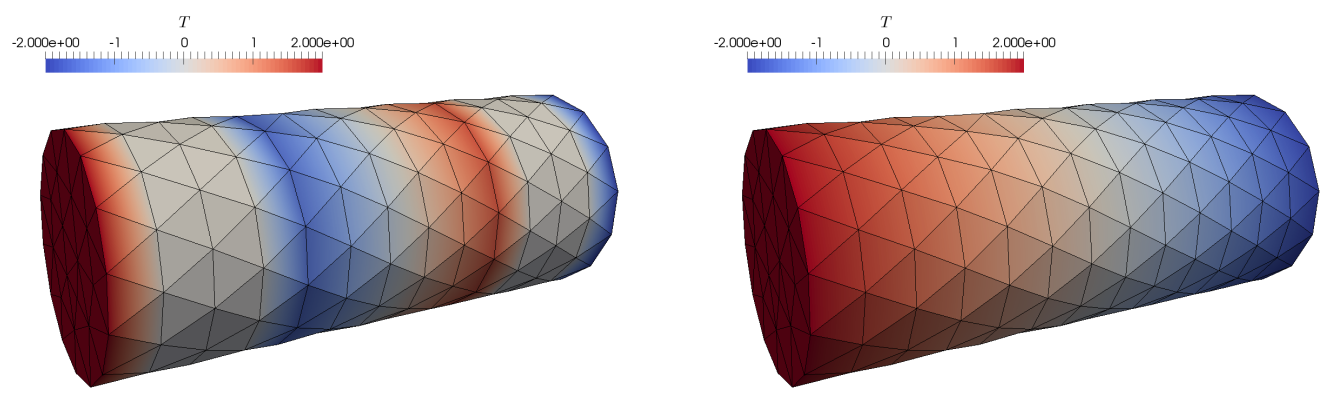

Figure 1: Initial and final snapshots of the temperature evolution.

\subsection{FE mesh handling and post-processing}

SfePy has no meshing capabilities besides several simple mesh generators (a block mesh generator, an open/closed cylinder mesh generator, a mesh generator from CT image data), but several operations like merging of matching meshes are supported. The FE mesh needs to be provided in a file in one of the supported formats, notably the legacy VTK format [26]. The results are stored in legacy VTK files, or in, usually in case of timedependent problems with many time steps, custom HDF5 [22] files. Many standard open-source tools can be used to display the VTK files, namely Paraview [23], or Mayavi [32], see data workflow of a simulation in Fig. 2. Mayavi is supported directly within $S f e P y$ via the postproc.py script. The extractor . py script is provided to extract/convert the HDF5 file content to VTK.

\subsection{Solvers}

SfePy provides and uses a unified interface to many standard codes, for example UMFPACK [18], MUMPS [3], PETSc [4], Pysparse [19] as well as the solvers available in SciPy. Various solver classes are supported: timestepping, nonlinear, linear, eigenvalue problem and optimization solvers. An automatically generated list of all the supported solvers can be found at the SfePy web site [43]. 


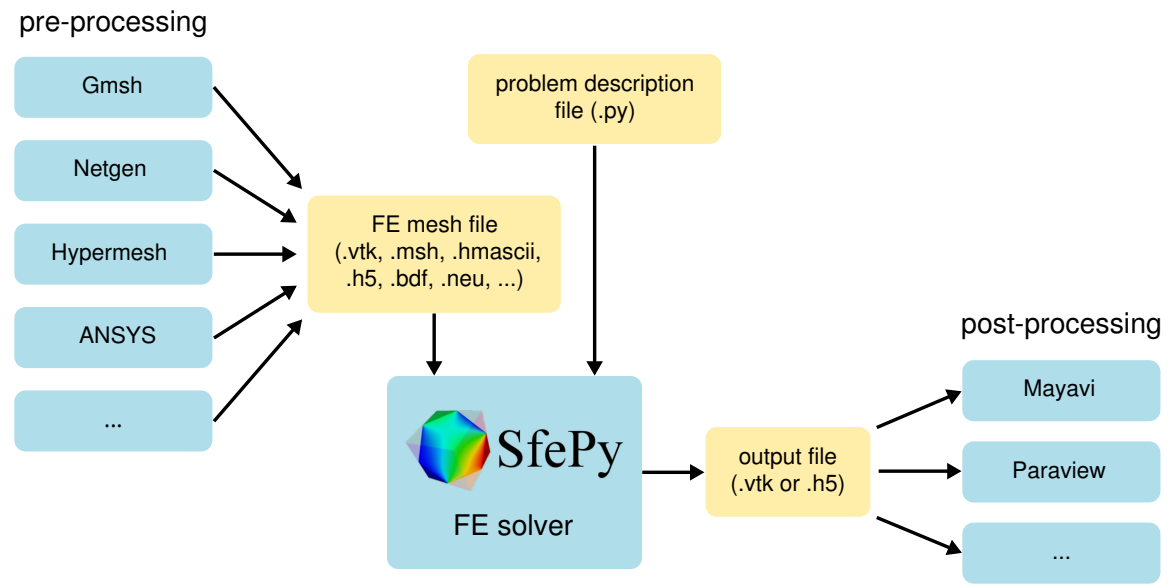

Figure 2: Data exchange between $S f e P y$ and external pre-processing and post-processing tools.

Besides external solvers, several solvers are implemented directly in $S f e P y$, for example:

- ts.simple: Implicit time stepping solver with a fixed time step, suitable also for quasistatic problems.

- ts.newmark, ts . bathe, ts.generalized_alpha: Solve elastodynamics problems by the Newmark, Bathe, generalized- $\alpha$ methods, respectively.

- nls.newton: The Newton nonlinear solver with a backtracking linesearch.

A typical problem solution, when using the declarative problem definition API, then involves calling a time-stepping solver that calls a nonlinear solver in each time step, which, in turn, calls a linear solver in the nonlinear iterations. A unified approach is used here: for stationary problems, a dummy time-stepping solver (ts.stationary) is automatically created. Similarly, a nonlinear solver is used to solve both the linear and non-linear problems. This simplifies imposing non-homogeneous Dirichlet boundary conditions: in the context of a nonlinear solver, the increment of the constrained DOFs is always zero and the non-zero boundary values can be ignored during the assembling. 


\subsection{Limitations}

The limitations can be split into two groups. The first group is related to limited number of developers and our research focus: certain features are missing, because they do not fall into our field of research (e.g. the vector finite elements). The limitations in the second group are more fundamental. Because the code relies on vectorization provided by NumPy, the code tries to work on all cells in a region in each operation: for example, all local finite element matrices are evaluated in a vectorized way into a single large NumPy array, and then assembled to a SciPy's sparse matrix. This places a restriction on a practically usable order of the basis function polynomials, especially for 3D hexahedrons, where orders greater than 4 are not practically usable. Using NumPy's arrays places another restriction: data homogeneity. So, for examples, the FE basis polynomial order has to be uniform over the whole (sub)domain where a field is defined. This is incompatible with an adaptive mesh refinement, especially the $h p$-adaptivity. Note that $S f e P y$ supports meshes with level-1 hanging nodes, so a limited $h$-adaptivity is partly possible.

\section{Homogenization engine}

In this section we briefly outline the approach to solving multiscale problems based on the theory of homogenization $[1,13]$. The main asset of the homogenization approach is that a homogenized model can take into account various details at the microstructure scale (topology, heterogeneous material parameters, etc.) without actually meshing those detailed features on a macroscopic domain, which would lead to an extremely large problem.

The homogenization engine is a feature that is in our opinion unique to $S f e P y$. It has been developed to allow an easy and flexible formulation of problems arising from use of the homogenization theory applied to strongly heterogeneous multiscale material models. Such models, as can be seen in the non-trivial example in Section 5, can have a complicated data flow and dependencies of various subproblems involved in the definition.

A typical homogenization procedure for a linear problem ${ }^{4}$ involves the following steps:

\footnotetext{
${ }^{4}$ The situation is much more complicated for nonlinear problems: a microproblem needs to be typically solved in every macroscopic integration point. This mode is also supported in $S f e P y$.
} 
1. Compute characteristic (corrector) functions by solving auxiliary corrector problems on a reference periodic cell domain that describes the microstructure (exactly or in a statistical sense).

2. Using the corrector functions, evaluate the homogenized coefficients. Those coefficients correspond to effective macroscopic properties of the material with the given microstructure as the microstructure characteristic scale tends to zero.

3. Solve the homogenized model with the obtained effective homogenized coefficients on a macroscopic domain.

There can be a number of the auxiliary corrector problems as well as the homogenized coefficients. The homogenization engine allows to describe their relationships and the dependencies among them and resolves the problems in a correct order automatically. A complete problem is described in one or more problem definition files using the declarative API, and data dependencies are described using Python dictionaries as a small domain-specific language.

The homogenization engine allows to solve microscopic subproblems and evaluate homogenized coefficients in parallel with use of either multithreading or multiprocessing features of a computer system. The distribution of microproblems between multiple threads or CPUs is governed by a function that puts the microproblems with resolved dependencies into the work queue, collects solved microscopic solutions and updates a dependency table according to the obtained results. Workers, i.e. threads or CPU cores, solve tasks from the work queue until it is empty. If the same microproblem needs to be solved multiple times with different parameters, typically for nonlinear problems, the total amount of microscopic tasks is divided into several chunks that are distributed to multiple workers. In the case of multiprocessing, the MPI library is used to communicate between computational nodes.

The parallel computation is crucial for nonlinear problems where microproblems have to be resolved in all macroscopic integration points in many time or iteration steps.

\section{Multiscale numerical simulation of piezoelectric structure}

The complex multiscale model, solved by the means of the homogenization method, and its implementation in $S f e P y$ are presented in this section. 


\subsection{Mathematical model of piezoelectric media}

We consider a porous piezoelectric medium which consists of a piezoelectric matrix, embedded metallic electrodes (conductors) and void inclusions. These components are arranged in a periodic lattice so that the medium can be generated by copies of the reference unit cell, see Fig.3. The mechanical behavior of such a structure can be described using the two-scale asymptotic homogenization method, see $[1,13]$. The quantities oscillating within the heterogeneous structure with the period equal to the size of the periodic unit are labelled by the superscript ${ }^{\varepsilon}$ in the subsequent text.

The mechanical properties of the piezoelectric solid are given by the following constitutive equations

$$
\begin{aligned}
& \sigma_{i j}^{\varepsilon}\left(\vec{u}^{\varepsilon}, \varphi^{\varepsilon}\right)=A_{i j k l}^{\varepsilon} e_{k l}\left(\vec{u}^{\varepsilon}\right)-g_{k i j}^{\varepsilon} E_{k}\left(\varphi^{\varepsilon}\right), \\
& D_{k}^{\varepsilon}\left(\vec{u}^{\varepsilon}, \varphi^{\varepsilon}\right)=g_{k i j}^{\varepsilon} e_{i j}\left(\vec{u}^{\varepsilon}\right)+d_{k l}^{\varepsilon} E_{l}\left(\varphi^{\varepsilon}\right),
\end{aligned}
$$

which express the dependencies of the Cauchy stress tensor $\boldsymbol{\sigma}^{\varepsilon}$ and the electric displacement $\vec{D}^{\varepsilon}$ on the strain tensor $\vec{e}\left(\vec{u}^{\varepsilon}\right)=\frac{1}{2}\left(\nabla \vec{u}^{\varepsilon}+\left(\nabla \vec{u}^{\varepsilon}\right)^{T}\right)$, where $\vec{u}^{\varepsilon}$ is the displacement field, and on the electric field $\vec{E}\left(\varphi^{\varepsilon}\right)=\nabla \varphi^{\varepsilon}$, where $\varphi^{\varepsilon}$ is the electric potential. On the right hand side of (1), we have the fourth-order elastic tensor $A_{i j k l}^{\varepsilon}\left(A_{i j k l}^{\varepsilon}=A_{k l i j}^{\varepsilon}=A_{j i l k}^{\varepsilon}\right)$, the third-order tensor $g_{k i j}^{\varepsilon}\left(g_{k i j}^{\varepsilon}=\right.$ $\left.g_{k j i}^{\varepsilon}\right)$, which couples mechanical and electric quantities, and the permeability tensor $d_{k l}^{\varepsilon}$.

The quasi-static problem of the piezoelectric medium is given by the following equilibrium equations

$$
\begin{array}{ll}
-\nabla \cdot \boldsymbol{\sigma}^{\varepsilon}\left(\vec{u}^{\varepsilon}, \varphi^{\varepsilon}\right)=\vec{f}^{\varepsilon}, & \text { in } \Omega_{m c}^{\varepsilon}, \\
-\nabla \cdot \vec{D}^{\varepsilon}\left(\vec{u}^{\varepsilon}, \varphi^{\varepsilon}\right)=q_{E}^{\varepsilon}, & \text { in } \Omega_{m}^{\varepsilon},
\end{array}
$$

and by the boundary conditions

$$
\begin{array}{cl}
\vec{n} \cdot \sigma^{\varepsilon}=\vec{h}^{\varepsilon} \text { on } \Gamma_{\sigma}^{\varepsilon}, \quad \vec{n} \cdot \vec{D}^{\varepsilon}=\varrho_{E}^{\varepsilon} \text { on } \Gamma_{\vec{D}}^{\varepsilon}, \\
\vec{u}^{\varepsilon}=\overline{\vec{u}} \text { on } \Gamma_{\vec{u}}^{\varepsilon}, \quad \varphi^{\varepsilon}=\bar{\varphi} \text { on } \Gamma_{\varphi}^{\varepsilon},
\end{array}
$$

where $\vec{f}, \vec{h}^{\varepsilon}$ are the volume and surface forces, $q_{E}^{\varepsilon}, \varrho_{E}^{\varepsilon}$ are the volume and surface charges and $\overline{\vec{u}}, \bar{\varphi}$ are the prescribed displacements and electric potential, respectively. The piezo-elastic medium occupies an open bounded region $\Omega \in \mathbb{R}^{3}$ which is decomposed into several non-overlapping parts: the piezoelectric elastic matrix $\Omega_{m}^{\varepsilon}$, conductive elastic parts $\Omega_{c}^{\varepsilon}=\bigcup_{k} \Omega_{c}^{k, \varepsilon}$ and 


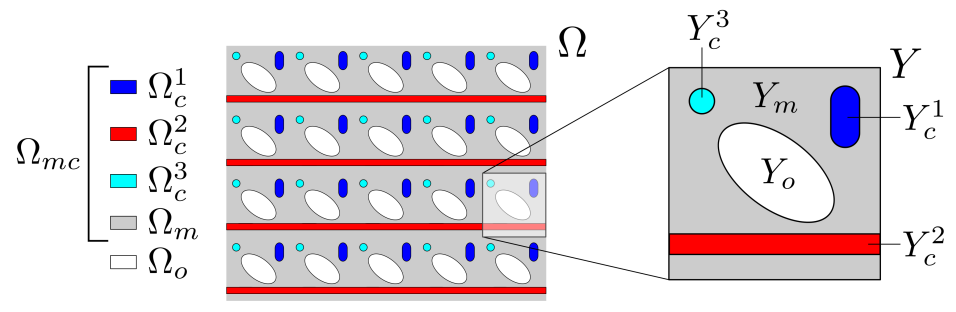

Figure 3: The scheme of the representative periodic cell decomposition and the generated periodic structure.

isolated void inclusions $\Omega_{o}^{\varepsilon}$, see Fig. 3. The elastic part, i.e. the matrix and the conductors, is denoted by $\Omega_{m c}^{\varepsilon}=\Omega_{m}^{\varepsilon} \cup \Omega_{c}^{\varepsilon}$.

The weak formulation of the problem stated above can be written as: Given volume and surface forces $\vec{f}^{\varepsilon}, \vec{h}^{\varepsilon}$ and volume and surface charges $q_{E}^{\varepsilon}$, $\varrho_{E}^{\varepsilon}$, find $\vec{u}^{\varepsilon} \in \mathcal{U}\left(\Omega_{m c}^{\varepsilon}\right), \varphi^{\varepsilon} \in \mathcal{V}\left(\Omega_{m}^{\varepsilon}\right)$ such that for all $\vec{v} \in \mathcal{U}_{0}\left(\Omega_{m c}^{\varepsilon}\right), \psi \in \mathcal{V}_{0}\left(\Omega_{m}^{\varepsilon}\right)$

$$
\begin{aligned}
& \int_{\Omega_{m}^{\varepsilon}}\left[\vec{A}^{\varepsilon} \vec{e}\left(\vec{u}^{\varepsilon}\right)-\left(\vec{g}^{\varepsilon}\right)^{T} \cdot \nabla \varphi^{\varepsilon}\right]: \vec{e}(\vec{v}) \mathrm{d} V+\int_{\Omega_{c}^{\varepsilon}}\left[\vec{A}^{\varepsilon} \vec{e}\left(\vec{u}^{\varepsilon}\right)\right]: \vec{e}(\vec{v}) \mathrm{d} V \\
& \quad=\int_{\Gamma_{\sigma}^{\varepsilon}} \vec{h} \cdot \vec{v} \mathrm{~d} S+\int_{\Omega_{m c}^{\varepsilon}} \vec{f}^{\varepsilon} \cdot \vec{v} \mathrm{~d} V, \\
& \int_{\Omega_{m}^{\varepsilon}}\left[\vec{g}^{\varepsilon}: \vec{e}\left(\vec{u}^{\varepsilon}\right)+\vec{d}^{\varepsilon} \cdot \nabla \varphi^{\varepsilon}\right] \cdot \nabla \psi \mathrm{d} V=\int_{\Gamma_{\vec{D}}^{\varepsilon}} \varrho_{E}^{\varepsilon} \psi \mathrm{d} S+\int_{\Omega_{m}^{\varepsilon}} q_{E}^{\varepsilon} \psi \mathrm{d} V .
\end{aligned}
$$

Symbols $\mathcal{U}, \mathcal{U}_{0}, \mathcal{V}, \mathcal{V}_{0}$ denote admissibility sets, where $\mathcal{U}_{0}, \mathcal{V}_{0}$ are the sets with zero trace on the Dirichlet boundary. Further details can be found in [36].

\subsection{Two-scale homogenization}

We apply the standard homogenization techniques, cf. [1] or [13], to the problem (4). It results in the limit model for $\varepsilon \longrightarrow 0$, where $\varepsilon$ is the scale parameter relating the microscopic and macroscopic length scales. The homogenization process leads to local microscopic problems, defined within a reference periodic cell, and to the global problem describing the behavior of the homogenized medium at the macroscopic level. The global problem involves the homogenized material coefficients which are evaluated using the solutions of the local problems. Due to linearity of the problem, the microscopic and macroscopic problems are decoupled. 
As we assume given potentials $\bar{\varphi}^{k}$ in each of the electrode networks, the dielectric properties must be appropriately rescaled in order to preserve the finite electric field for the limit $\varepsilon \longrightarrow 0: \vec{g}^{\varepsilon}=\varepsilon \overline{\vec{g}}, \vec{d}^{\varepsilon}=\varepsilon^{2} \overline{\vec{d}}$, cf. [36].

Local problems and homogenized coeffficients. The local microscopic responses of the piezoelectric structure are given by the following sub-problems which are solved within the periodic reference cell $Y$, see Fig. 3, that is decomposed similarly to the decomposition of domain $\Omega$ :

- Find $\boldsymbol{\omega}^{i j} \in \vec{H}_{\#}^{1}\left(Y_{m c}\right), \eta^{i j} \in H_{\# 0}^{1}\left(Y_{m}\right)$ such that for all $\vec{v} \in \vec{H}_{\#}^{1}\left(Y_{m c}\right)$, $\psi \in H_{\# 0}^{1}\left(Y_{m}\right)$ and for any $i, j=1,2,3$

$$
\begin{array}{r}
\int_{Y_{m c}}\left[\vec{A} \vec{e}\left(\boldsymbol{\omega}^{i j}+\boldsymbol{\Pi}^{i j}\right)\right]: \vec{e}(\vec{v}) \mathrm{d} V-\int_{Y_{m}}\left[\overrightarrow{\vec{g}}^{T} \cdot \nabla \eta^{i j}\right]: \vec{e}(\vec{v}) \mathrm{d} V=0, \\
\int_{Y_{m}}\left[\overline{\vec{g}}: \vec{e}\left(\boldsymbol{\omega}^{i j}+\boldsymbol{\Pi}^{i j}\right)+\overline{\vec{d}} \cdot \nabla \eta^{i j}\right] \cdot \nabla \psi \mathrm{d} V=0,
\end{array}
$$

where $\Pi_{k}^{i j}=y_{j} \delta_{i k}$.

- Find $\hat{\boldsymbol{\omega}}^{k} \in \vec{H}_{\#}^{1}\left(Y_{m c}\right), \hat{\eta}^{k} \in H_{\# 0, k}^{1}\left(Y_{m}\right)$ such that for all $\vec{v} \in \vec{H}_{\#}^{1}\left(Y_{m c}\right), \psi \in$ $H_{\# 0}^{1}\left(Y_{m}\right)$ and for any $k=1,2, \ldots, k^{c}$ ( $k^{c}$ is the number of conductors)

$$
\begin{array}{r}
\int_{Y_{m c}}\left[\vec{A} \vec{e}\left(\hat{\boldsymbol{\omega}}^{k}\right)\right]: \vec{e}(\vec{v}) \mathrm{d} V-\int_{Y_{m}}\left[\overline{\vec{g}}^{T} \cdot \nabla \hat{\eta}^{k}\right]: \vec{e}(\vec{v}) \mathrm{d} V=0, \\
\int_{Y_{m}}\left[\overline{\vec{g}}: \vec{e}\left(\hat{\boldsymbol{\omega}}^{k}\right)+\overline{\vec{d}} \cdot \nabla \hat{\eta}^{k}\right] \cdot \nabla \psi \mathrm{d} V=0 .
\end{array}
$$

The microscopic sub-problems are solved with the periodic boundary conditions and $\hat{\eta}^{k}=\delta_{k i}$ on $\Gamma_{m c}^{i}$ for $i=1,2, \ldots, k^{c}, \Gamma_{m c}^{i}=\overline{Y_{m}} \cap \overline{Y_{c}^{i}}$ is the interface between the matrix part $Y_{m}$ and $i$-th conductor $Y_{c}^{i}$. By $\vec{H}_{\#}^{1}$ we refer to the Sobolev space of $Y$-periodic functions, $H_{\# 0, k}^{1}$ reflects the above mentioned interface condition on $\Gamma_{m c}^{i}$ and $H_{\# 0}^{1}$ is the set of functions which are equal to zero on $\Gamma_{m c}$.

With the characteristic responses $\boldsymbol{\omega}^{i j}, \eta^{i j}$ and $\hat{\boldsymbol{\omega}}^{k}, \hat{\eta}^{k}$ obtained by solving (5) and (6), the homogenized material coefficients $\vec{A}^{H}$ and $\vec{P}^{H, k}$ can be 
evaluated using the following expressions:

$$
\begin{aligned}
& A_{i j k l}^{H}=\frac{1}{|Y|}\left[\int_{Y_{m c}}\left[\vec{A} \vec{e}\left(\boldsymbol{\omega}^{i j}+\boldsymbol{\Pi}^{i j}\right)\right]: \vec{e}\left(\boldsymbol{\omega}^{k l}+\boldsymbol{\Pi}^{k l}\right) \mathrm{d} V+\int_{Y_{m}} \overline{\vec{d} \nabla} \eta^{i j} \cdot \nabla \eta^{k l} \mathrm{~d} V\right] \\
& P_{i j}^{H, k}=\frac{1}{|Y|}\left[\int_{Y_{m c}}\left[\vec{A} \vec{e}\left(\hat{\boldsymbol{\omega}}^{k}\right)\right]: \vec{e}\left(\boldsymbol{\Pi}^{i j}\right) \mathrm{d} V-\int_{Y_{m}}\left[\overline{\vec{g}}: \vec{e}\left(\boldsymbol{\Pi}^{i j}\right)\right] \cdot \nabla \hat{\eta}^{k} \mathrm{~d} V\right] .
\end{aligned}
$$

Macroscopic problem. The global macroscopic problem is defined in terms of the homogenized coefficients as: Find the macroscopic displacements $\vec{u}^{0} \in$ $\mathcal{U}(\Omega)$ such that for all $\vec{v}^{0} \in \mathcal{U}_{0}(\Omega)$

$$
\int_{\Omega}\left[\vec{A}^{H} \vec{e}\left(\vec{u}^{0}\right)\right]: \vec{e}(\vec{v}) \mathrm{d} V=-\int_{\Omega} \vec{e}(\vec{v}): \sum_{k} \vec{P}^{H, k} \bar{\varphi}^{k} \mathrm{~d} V .
$$

We assumed that $\varrho_{E}=0$, otherwise we would need an extra coefficient related to the surface charge, see [36].

\subsection{Numerical simulation}

In this section we illustrate the use of SfePy's homogenization engine in the following setting. The macroscopic problem described by (8) is solved in the domain $\Omega$, depicted in Fig. 4 right, that is fixed at its left face $\left(u_{i}^{0}=0\right.$ for $i=1,2,3$ on $\left.\Gamma_{\text {left }}\right)$. No volume and surface forces or charges are applied and the deformation of the macroscopic sample is invoked only by the prescribed electrical potential $\bar{\varphi}= \pm 10^{4} \mathrm{~V}$ in the two embedded conductor networks. The geometry of the representative volume element, which is used to solve the microscopic problems (5), (6), is depicted in Fig. 4 left. The material parameters of the piezoelectric elastic matrix, made of barium-titanite, and metallic conductors are summarized in Table 1.

The results of the multiscale numerical simulation are shown in Figs. 5, 6. The macroscopic strain field and the deformed macroscopic sample (deformation scaled by factor 100) are presented in Fig. 5. Using the macroscopic solution and the characteristic responses, we can reconstruct the fields at the microscopic level for a given finite size $\varepsilon_{0}$ in a chosen part of the macroscopic domain. The reconstructed strain field and the deformed microstructure (deformation scaled by factor 10) are shown in Fig. 6 left, the reconstructed electric field is depicted in Fig. 6 right. See [36] for comparison of accuracy of the reconstructed solutions with a full, much more demanding, simulation. In 


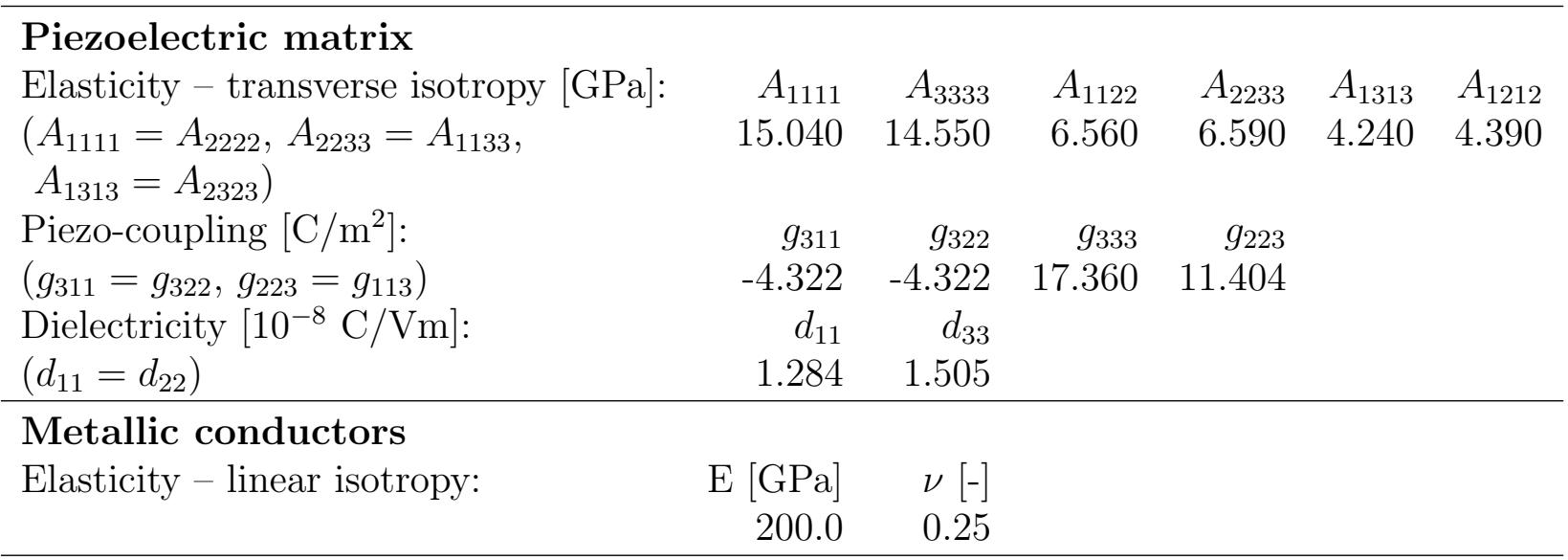

Table 1: Properties of the piezoelectric matrix and metallic conductors.
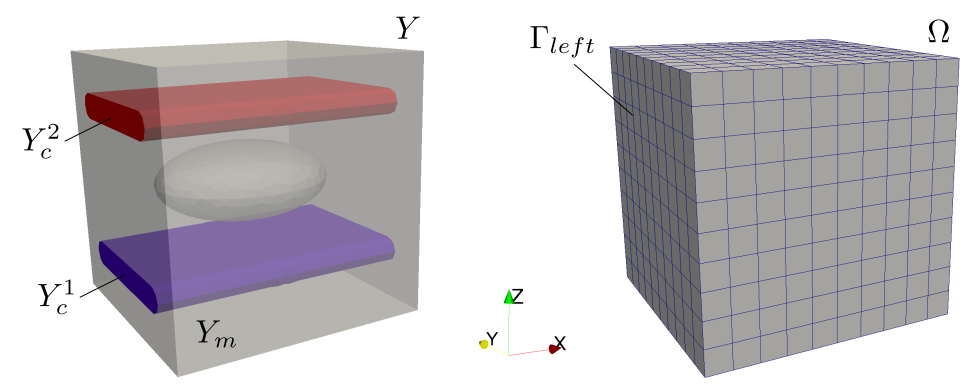

Figure 4: Left: the geometry of the reference periodic cell $Y$; Right: the macroscopic domain $\Omega$.

the above article, the full (reference) simulation has approximately $4.5 \times 10^{5}$ degrees of freedom while the microscopic problem has only 741 DOFs and the macroscopic problem 577 DOFs.

\subsection{Multiscale analysis in SfePy}

The linear multiscale analysis defined above is performed in $S f e P y$ in two steps:

1. The local microscopic sub-problems (5), (6) are solved using the homogenization engine, see Section 4. The engine is also used to evaluate the homogenized coefficients according to (7).

2. The global macroscopic problem (8) is solved, the known homogenized coefficients are employed. 


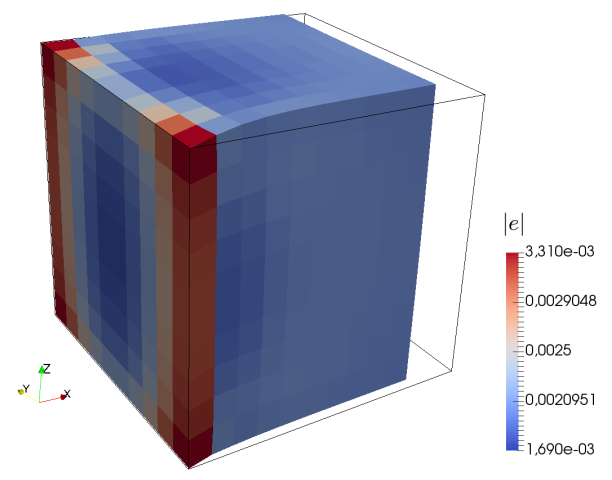

Figure 5: The deformed macroscopic sample (deformation scaled by factor 100) and the magnitude of macroscopic strain field.
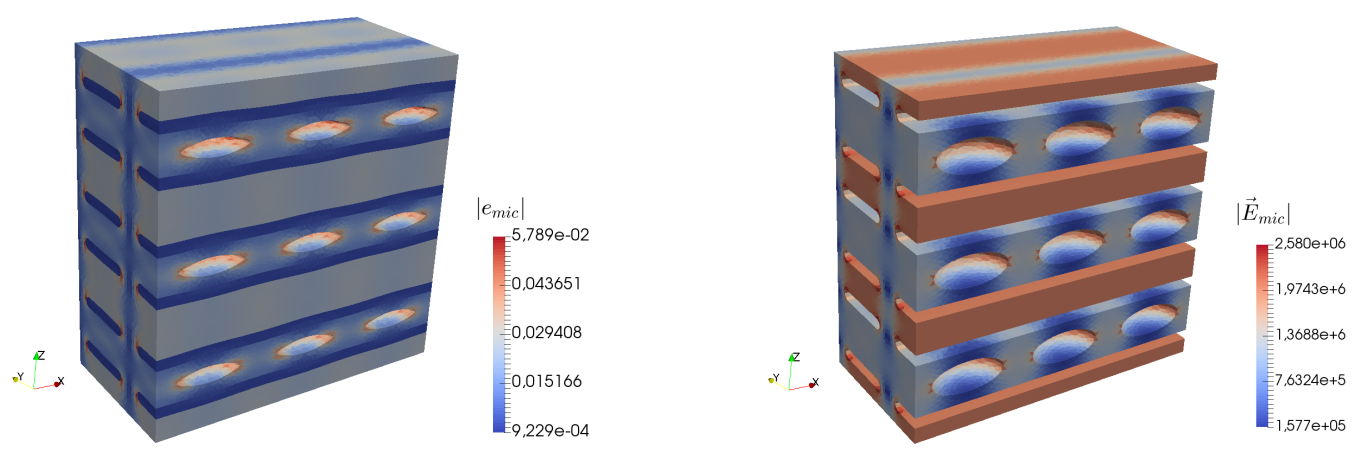

Figure 6: Left: the deformed microscopic structure (deformation scaled by factor 10) and the magnitude of reconstructed strain field; Right: the magnitude of the reconstructed electric field. 
The definition of the global problem can be done in the similiar way as in the simple heat conduction example presented in Section 3.4. The macroscopic equation in the declarative API attains the form

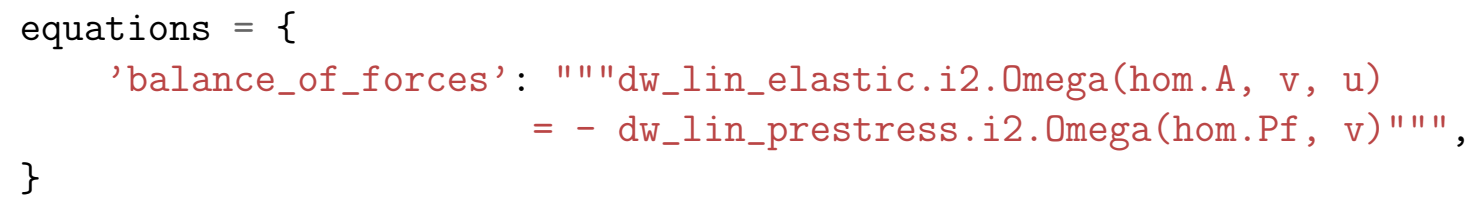

where hom. A stands for the homogenized coefficients $\vec{A}^{H}$ and hom.Pf is equal to $\sum_{k} \vec{P}^{H, k} \bar{\varphi}^{k}$, i.e. the sum of the coefficients $\vec{P}^{H, k}$ multiplied by the prescribed electrical potentials $\bar{\varphi}^{k}$. The homogenized material hom is declared as a function, which calls the homogenization engine (via SfePy's built-in function get_homog_coefs_linear () in the following code) and returns the calculated homogenized parameters. In the case of a linear problem, the same values are valid in all quadrature points of a macroscopic domain (coors argument in the function below).

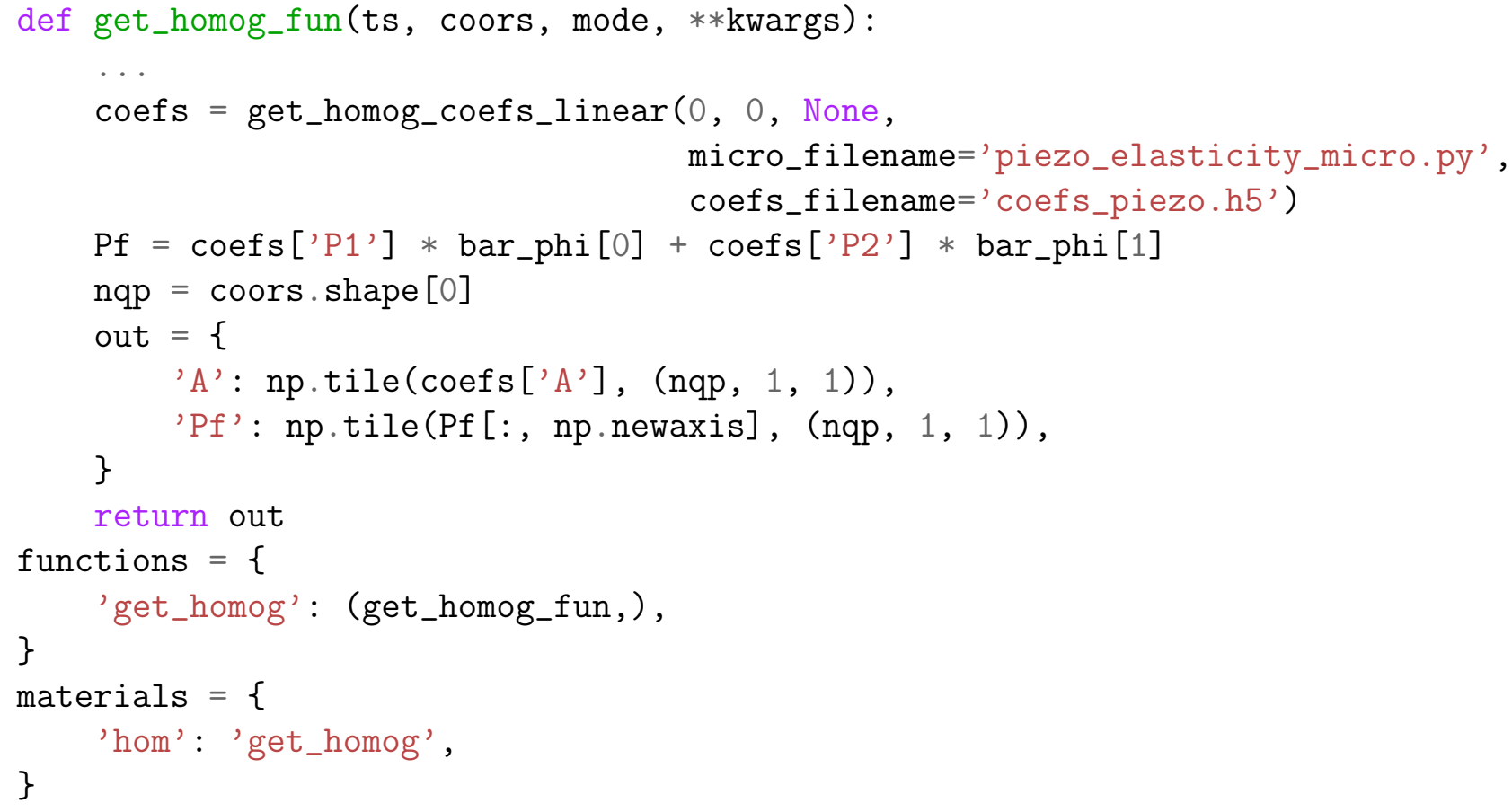

To define the microscopic sub-problems which are solved by the homogenization engine the following fields and variables are needed: 


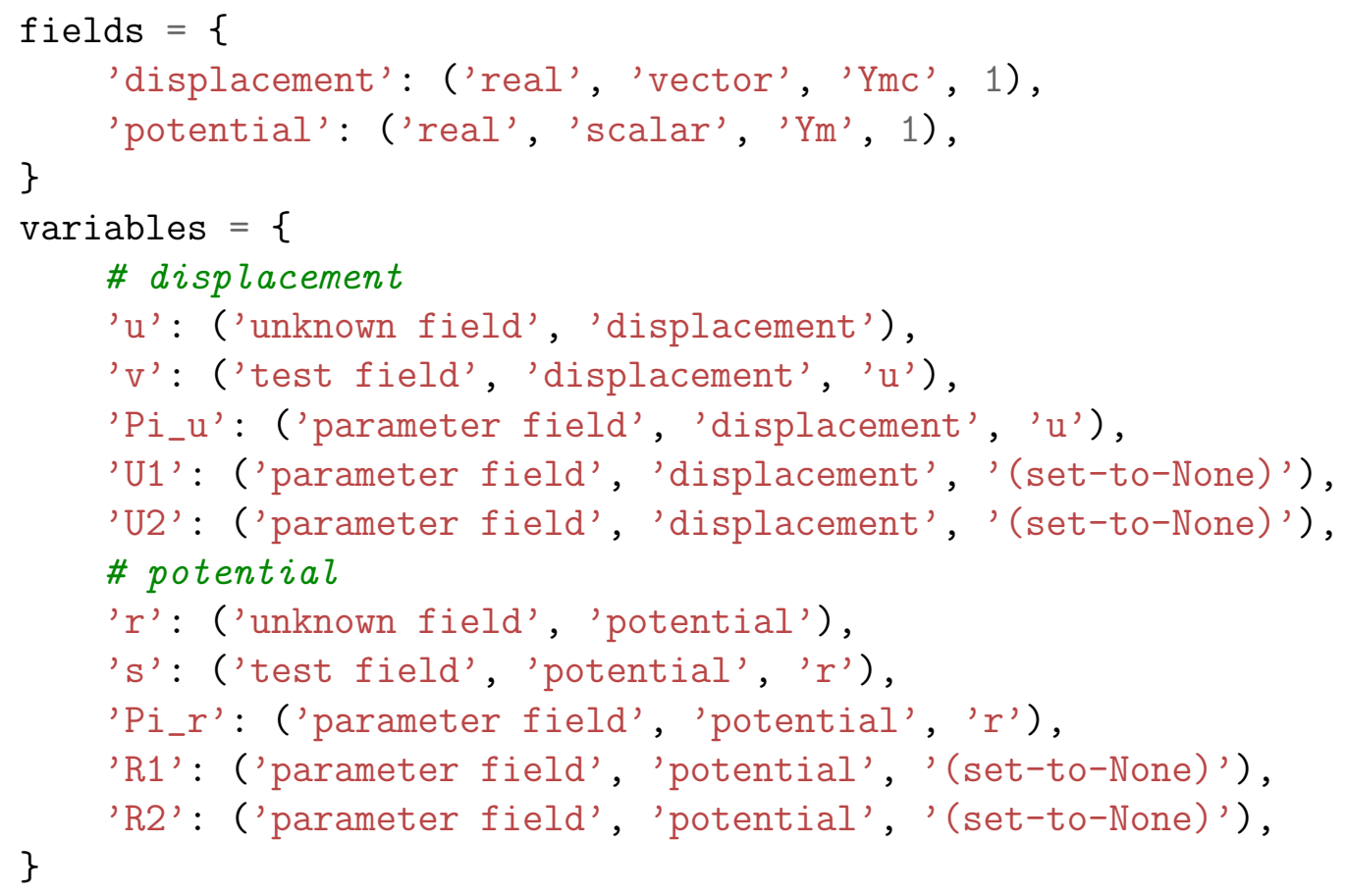

The material parameters of the elastic matrix $\left(Y_{m}\right)$ and the metalic conductors $\left(Y_{c}\right)$ are defined as follows, see Table 1:

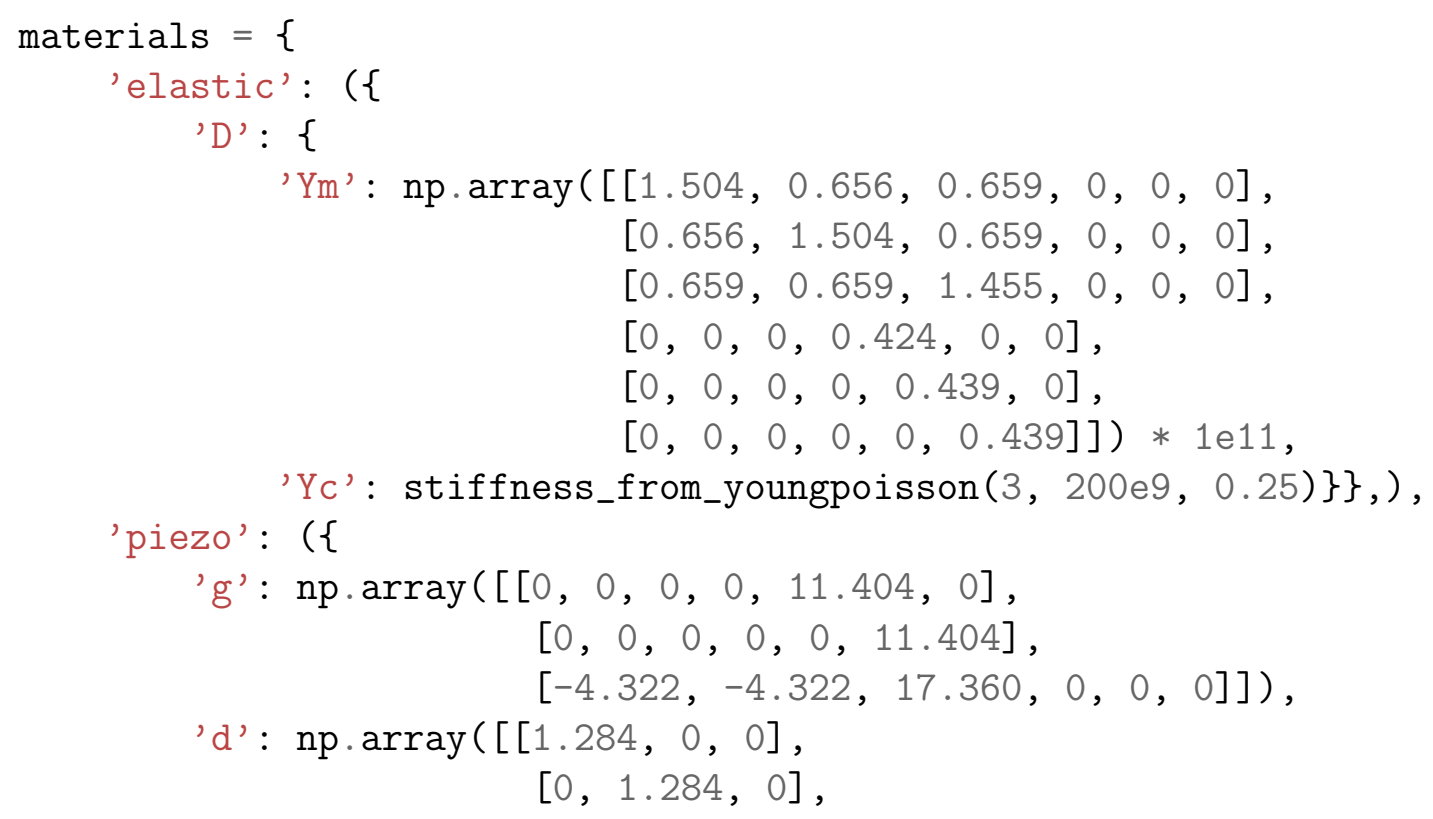




$$
[0,0,1.505]]) * 1 \mathrm{e}-8\},)
$$

\}

The homogenized coefficients $\vec{A}^{H}$ can be introduced as

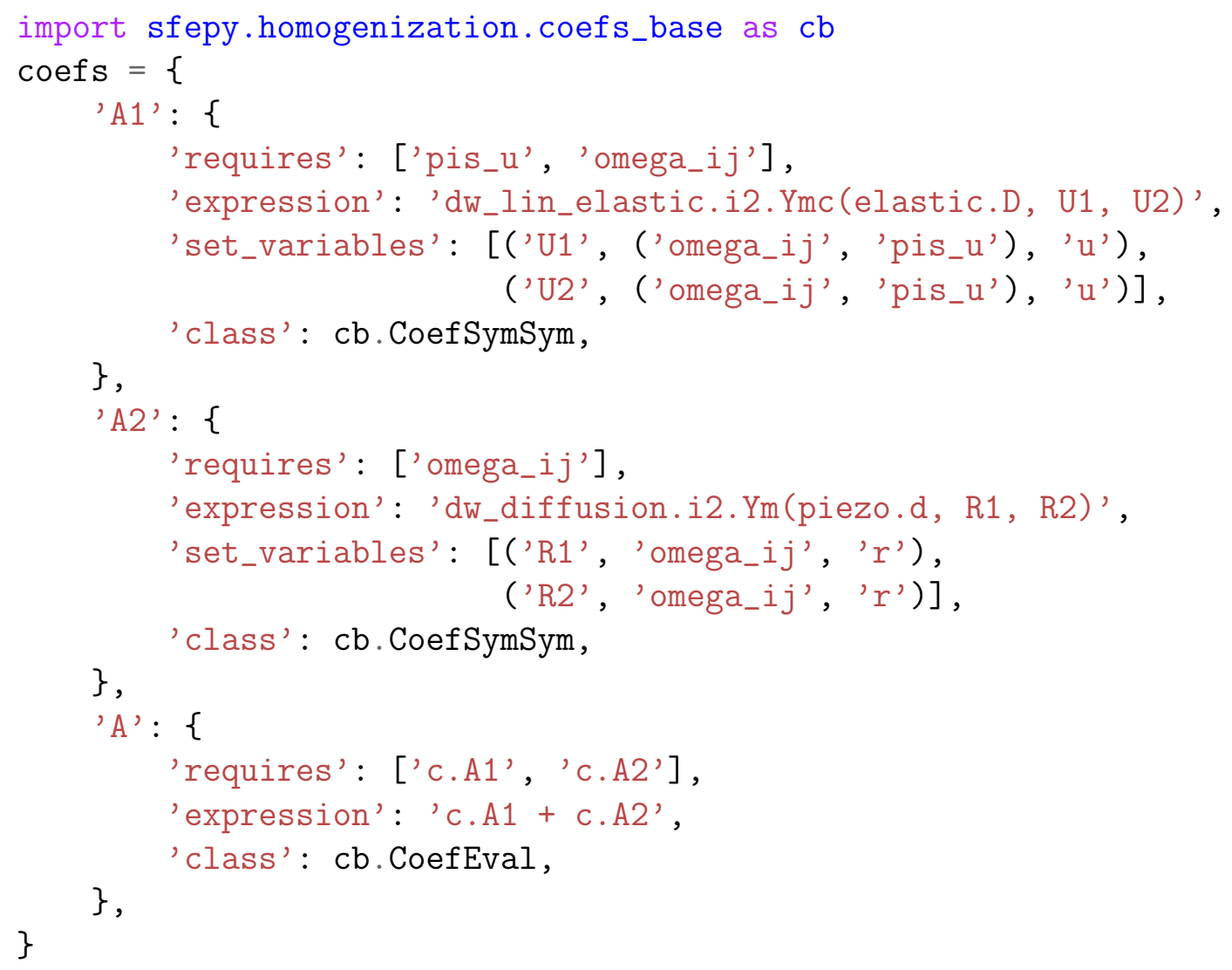

where we follow the expression $\left(7_{1}\right)$ which consists of two integrals over domains $Y_{m c}$ (matrix + conductors) and $Y_{c}$ (conductors). The definition of each coefficient has these parts: requires - the names of correctors needed for evaluation, expression - the expression to be evaluated, class - the coefficient class; it determines the way of evaluation and the resulting matrix/array shape. In our case, the class of A1 and A2 is CoefSymSym: it means that the resulting coefficients are the fourth-order tensors in the symetric storage, e.g. sym $\times$ sym matrices, where sym is the number of components in a symmetric stress/strain vector. Class CoefEval is used to evaluate a simple mathematical expression, in our example, the summation of $\mathrm{A} 1$, A2. In set_variables section we say how to substitute the correctors into 
the variables employed in the expression. For example, the code ('U1', ('omega_ij', 'pis_u'), 'u') is interpretted as: $U 1=\boldsymbol{\omega}^{K}+\boldsymbol{\Pi}^{K}$, where $\boldsymbol{\omega}^{K}$ is stored in omega_ij['u'], $\boldsymbol{\Pi}^{K}$ in pis_u['u'] and $K$ is the multiindex attaining 11,22,33,12,13,23 for a $3 \mathrm{D}$ problem because of the used CoefSymSym class. In a similar way, the coefficients $\vec{P}^{H, 1}$ can be introduced as

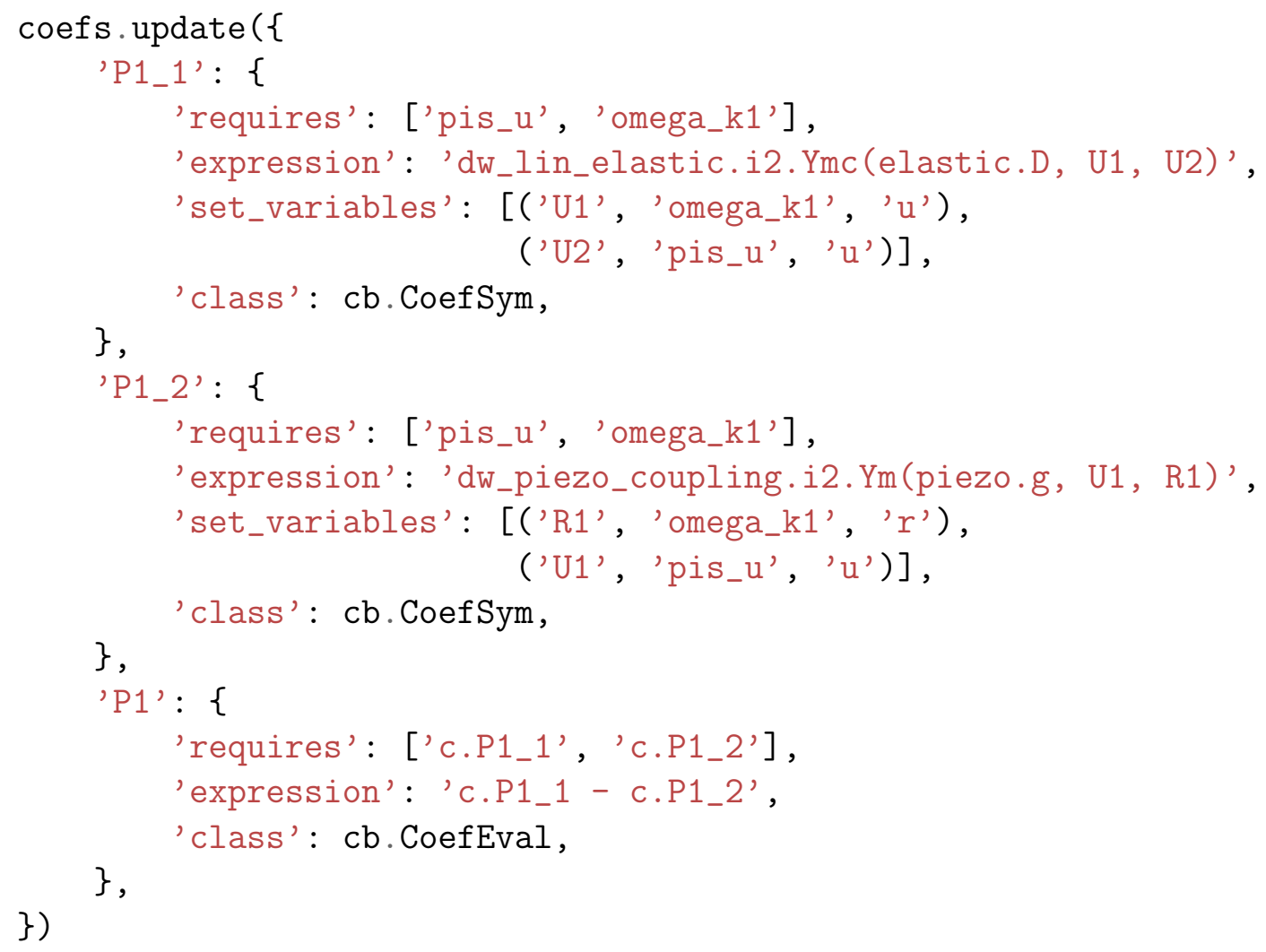

Here, the CoefSym class is employed due to the second-order coefficient which can be represented as a vector with dimension sym.

The required correctors omega_ij, see (5), and pis_u are defined as follows:

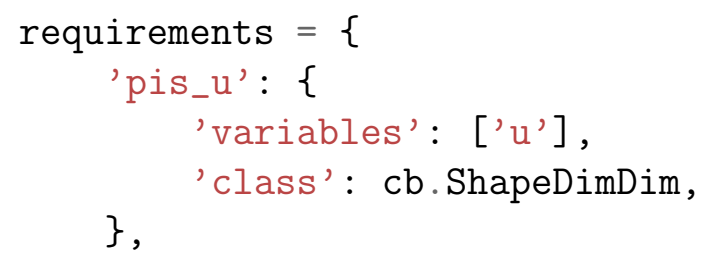




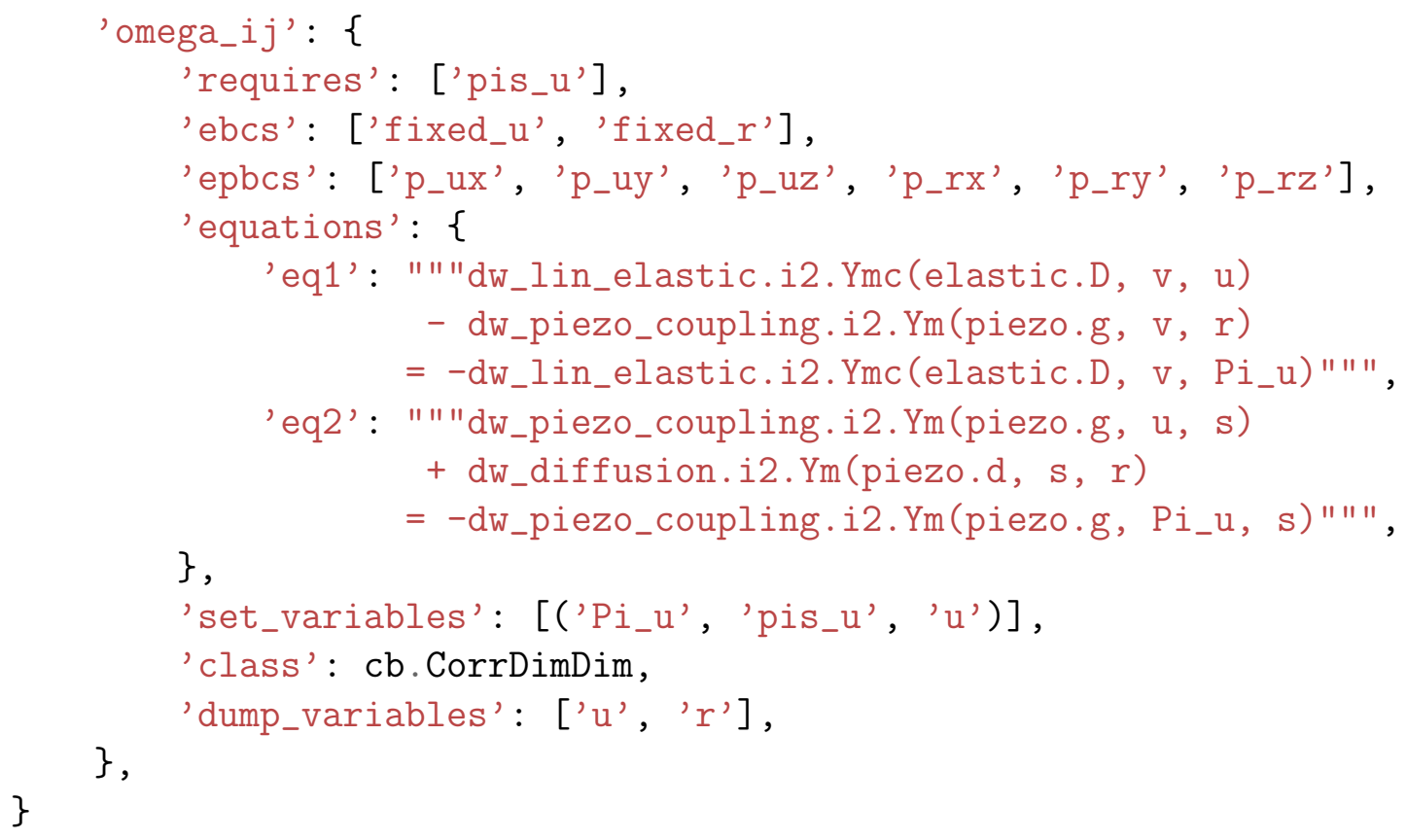

The class ShapeDimDim is used to define the symbol $\Pi_{k}^{i j}=y_{j} \delta_{i k}$ and CorrDimDim ensures the corrector with $\operatorname{dim} \times \operatorname{dim}$ components, dim is the space dimension. Note that a corrector can also depend on another corrector as in the code above, where pis_u is required to solve omega_ij. The correctors introduced in (6) can be defined in $S f e P y$ as

requirements . update $(\{$

'omega_k1: \{

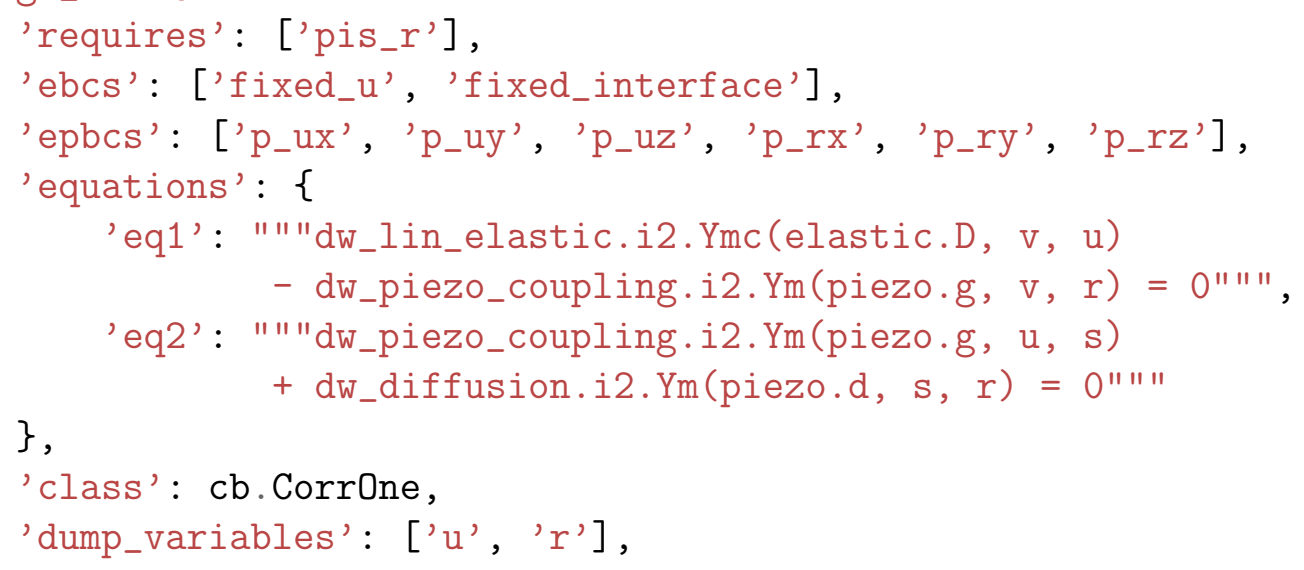


The class CoefOne corresponds to the scalar corrector function. The correctors are solved with the periodic boundary conditions defined in the lines with the keyword epbcs and the Dirichlet (essential) boundary conditions defined in the lines with the keyword ebcs.

The multiscale simulation can be run by calling the simple.py script, see Section 3.5, with the name of the description file for the macroscopic problem as a script parameter. The script runs the simulation at the macroscopic level and invokes the homogenization engine through the material function. The full sources of this example can be found in the SfePy package in examples/multiphysics/: piezo_elasticity_macro.py defines the macroscopic problem, and piezo_elasticity_micro.py defines the computations on the reference periodic cell of the microstructure. For the version of the sources used in this article see [8].

\section{Conclusion}

We introduced the open source finite element package $S f e P y$, a code written (mostly) in Python for solving various kinds of problems described by partial differential equations and discretized by the finite element method. The design of the code was discussed and illustrated using a simple heat conduction example.

Special attention was devoted to the description of the SfePy's homogenization engine, a sub-package for defining complex multiscale problems. This feature was introduced in a tutorial-like form using a multiscale numerical simulation of a piezoelectric structure.

For the complete code of the examples presented, together with the required FE meshes and the 2018.3 version of $S f e P y$, see [8]. Further documentation and many more examples of $S f e P y$ use can be found on the project's web site [43].

Acknowledgment. This work was supported by the projects GA17-12925S and GA16-03823S of the Czech Science Foundation and by the project LO1506 of the Czech Ministry of Education, Youth and Sports.

[1] Allaire G (1992) Homogenization and two-scale convergence. SIAM J Math Anal 23:1482-1518, DOI 10.1137/0523084

[2] Alnaes MS, Blechta J, Hake J, Johansson A, Kehlet B, Logg A, Richardson C, Ring J, Rognes ME, Wells GN (2015) The fenics project version 1.5. Arch Numer Software 3, DOI 10.11588/ans.2015.100.20553 
[3] Amaya M, Morten JP, Boman L (2016) A low-rank approximation for large-scale 3d controlled-source electromagnetic gauss-newton inversion. Geophysics 81(3), DOI 10.1190/geo2015-0079.1

[4] Balay S, Abhyankar S, Adams M, Brown J, Brune P, Buschelman K, Dalcin L, Dener A, Eijkhout V, Gropp W, Kaushik D, Knepley M, May D, Curfman McInnes L, Mills R, Munson T, Rupp K, Sanan P, Smith B, Zampini S, Zhang H, Zhang H (2018) PETSc users manual. Tech. Rep. ANL-95/11 - Revision 3.10, Argonne National Laboratory, URL http://www.mcs.anl.gov/petsc, accessed 25 September 2018

[5] Borden MJ, Scott MA, Evans JA, Hughes TJR (2011) Isogeometric finite element data structures based on Bezier extraction of NURBS. Int J Numer Meth Engng 87:15-47, DOI 10.1002/nme.2968

[6] Bradshaw R, Behnel S, Seljebotn DS, Ewing G, et al. (2018) The Cython compiler. Http://cython.org, Accessed 25 September 2018

[7] Cimrman R (2014) Enhancing sfepy with isogeometric analysis. arXiv:14126407 [cs, math] URL http://arxiv.org/abs/1412.6407, arXiv: 1412.6407

[8] Cimrman R, Lukeš V (2018) SfePy 2018.3 sources and heat conduction examples demonstrating declarative and imperative APIs of SfePy. https://zenodo.org/record/1434071, DOI 10.5281/zenodo.1434071

[9] Cimrman R, Rohan E (2007) On modelling the parallel diffusion flow in deforming porous media. Math Comput Simul 76(1-3):34-43, DOI 10.1016/j.matcom.2007.01.034

[10] Cimrman R, Rohan E (2010) Two-scale modeling of tissue perfusion problem using homogenization of dual porous media. Int J Multiscale Com 8(1):81-102, DOI 10.1615/IntJMultCompEng.v8.i1.70

[11] Cimrman R, Novák M, Kolman R, Tưma M, Plešek P, Vackáŕ J (2018) Convergence study of isogeometric analysis based on bézier extraction in electronic structure calculations. Appl Math Comput 319:138-152, DOI 10.1016/j.amc.2017.02.023 
[12] Cimrman R, Novák M, Kolman R, Tůma M, Vackáŕ J (2018) Isogeometric analysis in electronic structure calculations. MathComput Simulat 145:125-135, DOI 10.1016/j.matcom.2016.05.011

[13] Cioranescu D, Donato P (1999) An introduction to homogenization. No. 17 in Oxford Lecture Series in Mathematics and its Applications, Oxford University Press, Oxford

[14] Cottrell JA, Hughes TJR, Bazilevs Y (2009) Isogeometric Analysis: Toward Integration of CAD and FEA. John Wiley \& Sons

[15] Dalcin L, Paz R, Kler P, Cosimo A (2011) Parallel distributed computing using python. Adv Water Resour 34(9):1124-1139, DOI 10.1016/j. advwatres.2011.04.013

[16] Dalcin L, Collier N, Vignal P, Cortes A, Calo V (2016) Petiga: A framework for high-performance isogeometric analysis. Comput Method Appl M 308(C), DOI 10.1016/j.cma.2016.05.011

[17] Dalcin LD, Paz RR, Kler PA, Cosimo A (2011) Parallel distributed computing using python. Adv Water Resour 34(9):1124-1139, DOI 10. 1016/j.advwatres.2011.04.013

[18] Davis TA (2004) Algorithm 832: UMFPACK, an unsymmetric-pattern multifrontal method. ACM T Math Software 30(2):196-199, DOI 10. $1145 / 992200.992206$

[19] Geus R, Wheeler D, Orban D (2018) Pysparse documentation. Http://pysparse.sourceforge.net, Accessed 25 September 2018

[20] git (2018) The git project web site. Https://git-scm.com, Accessed 25 September 2018

[21] github (2018) Github web site. Https://github.com, Accessed 25 September 2018

[22] Group TH (2018) Hierarchical data format version 5. Http://www.hdfgroup.org/HDF5, Accessed 25 September 2018

[23] Henderson A (2007) ParaView Guide, A Parallel Visualization Application. Kitware Inc. 
[24] Hunter JD (2007) Matplotlib: A 2d graphics environment. Comput Sci Eng 9(3):90-95, DOI 10.1109/MCSE.2007.55

[25] Jones E, Oliphant TE, Peterson P, et al. (2018) SciPy: Open source scientific tools for Python. Http://www.scipy.org, Accessed 25 September 2018

[26] Kitware, Inc (2010) The Visualization Toolkit User's Guide. Kitware, Inc. publishers., iSBN 1-930934-18-1

[27] Kochová P, Cimrman R, Stengl M, Oštádal B, Tonar Z (2015) A mathematical model of the carp heart ventricle during the cardiac cycle. J Theor Bio 373:12-25, DOI 10.1016/j.jtbi.2015.03.014

[28] Logg A (2009) Efficient representation of computational meshes. Int J Comput Sci Eng 4(4):283-295, DOI 10.1504/IJCSE.2009.029164

[29] Meurer A, Smith CP, Paprocki M, Čertík O, Kirpichev SB, Rocklin M, Kumar A, Ivanov S, Moore JK, Singh S, Rathnayake T, Vig S, Granger BE, Muller RP, Bonazzi F, Gupta H, Vats S, Johansson F, Pedregosa F, Curry MJ, Terrel AR, Roučka Š, Saboo A, Fernando I, Kulal S, Cimrman R, Scopatz A (2017) Sympy: symbolic computing in python. PeerJ Comput Sci 3:e103, DOI 10.7717/peerj-cs.103

[30] Oliphant TE (2007) Python for scientific computing. Comput Sci Eng $9(3): 10-20$

[31] pytables (2018) Pytables web site. Https://www.pytables.org, Accessed 25 September 2018

[32] Ramachandran P, Varoquaux G (2011) Mayavi: 3d visualization of scientific data. Comput Sci Eng 13(2):40-51, DOI 10.1109/MCSE.2011.35

[33] Rathgeber F, Ham D, Mitchell L, Lange M, Luporini F, Mcrae A, Bercea GT, Markall G, Kelly P (2016) Firedrake: Automating the finite element method by composing abstractions. ACM T Math Software 43(3):24:124:27, DOI 10.1145/2998441

[34] Rohan E, Cimrman R (2012) Multiscale fe simulation of diffusiondeformation processes in homogenized dual-porous media. Math Comput Simul 82(10):1744-1772, DOI 10.1016/j.matcom.2011.02.011 
[35] Rohan E, Lukeš V (2010) Homogenization of the acoustic transmission through a perforated layer. J Comput Appl Math 234(6):1876-1885, DOI 10.1016/j.cam.2009.08.059

[36] Rohan E, Lukeš V (2018) Homogenization of the fluid-saturated piezoelectric porous media. Int J Solids Struct 147:110-125, DOI 10.1016/j. ijsolstr.2018.05.017

[37] Rohan E, Miara B (2011) Band gaps and vibration of strongly heterogeneous reissner-mindlin elastic plates. C R Math 349(13-14):777-781, DOI 10.1016/j.crma.2011.05.013

[38] Rohan E, Cimrman R, Naili S, Lemaire T (2009) Multiscale modelling of compact bone based on homogenization of double porous medium. In: Computational Plasticity X - Fundamentals and Applications

[39] Rohan E, Miara B, Seifrt F (2009) Numerical simulation of acoustic band gaps in homogenized elastic composites. Int J Eng Sci 47(4):573594, DOI 10.1016/j.ijengsci.2008.12.003

[40] Rohan E, Naili S, Cimrman R, Lemaire T (2012) Hierarchical homogenization of fluid saturated porous solid with multiple porosity scales. C R Mecanique 340(10):688-694, DOI 10.1016/j.crme.2012.10.022

[41] Rohan E, Naili S, Cimrman R, Lemaire T (2012) Multiscale modeling of a fluid saturated medium with double porosity: Relevance to the compact bone. J Mech Phys Solids 60(5):857-881, DOI 10.1016/j.jmps. 2012.01.013

[42] scikit-umfpack (2018) skikit-umfpack web site. Https://github.com/scikit-umfpack/scikit-umfpack, Accessed 25 September 2018

[43] sfepy (2018) The SfePy project web site. Http://sfepy.org, Accessed 25 September 2018

[44] Solin P, Segeth K, Dolezel I (2003) Higher-Order Finite Element Methods. Chapman \& Hall / CRC Press

[45] sphinx (2018) Sphinx web site. Http://www.sphinx-doc.org, Accessed 25 September 2018 
[46] travis-ci (2018) Travis-ci web site. Https://travis-ci.org, Accessed 25 September 2018

[47] Vackář J, Čertík O, Cimrman R, Novák M, Šipr O, Plešek J (2011) Advances in the Theory of Quantum Systems in Chemistry and Physics, Prog. T. Chem, vol 22, Springer, chap Finite Element Method in Density Functional Theory Electronic Structure Calculations, pp 199-217. DOI 10.1007/978-94-007-2076-3\_12

[48] Wu B, Du X, Tan H (1996) A three-dimensional fe nonlinear analysis of membranes. Comput Struct 59(4):601-605, DOI 10.1016/0045-7949(95) 00283-9

[49] Zemčík R, Rolfes R, Rose M, Tessmer J (2006) High-performance 4node shell element with piezoelectric coupling. Mech Adv Mater Struct 13(5):393-401, DOI 10.1080/15376490600777657 